\title{
Study on Office Building Window Design Strategy in Severe Cold Region Based on Daylight Comfort
}

\author{
Wang Zhao*, Yin Qing \\ School of Architecture, Harbin Institute of Technology, Harbin, China \\ Email address: \\ 1512391859@qq.com (Wang Zhao), hityin@126.com (Yin Qing) \\ ${ }^{*}$ Corresponding author
}

\section{To cite this article:}

Wang Zhao, Yin Qing. Study on Office Building Window Design Strategy in Severe Cold Region Based on Daylight Comfort. Science Discovery. Vol. 5, No. 7, 2017, pp. 572-578. doi: 10.11648/j.sd.20170507.27

Received: August 20, 2017; Accepted: December 19, 2017; Published: December 29, 2017

\begin{abstract}
Effective and reasonable daylight can create a comfortable and healthy lighting environment, and significantly affect the quality of indoor light environment in office buildings. Reasonable architectural form design can improve indoor light environment quality and visual comfort degree, and has great significance. Combining with light climate characteristics in severe cold region, the study based on parametric model, aims to analyze the influence degree of the window-wall ratio, glass transmittance and window shape on lighting comfort of westward office buildings. Taking a westward office building in Harbin as an example, Rhino \& Grasshopper is used to establish the parametric simulation model. Using different conditions of Window-Wall Ratio (WWR), Window Height (WH) and Height-Width Ratio (H/W) as variables to calculate the value of Daylight Factors (DF) and Daylight Discomfort Probability (DGP) through the control variable method. Adopting the regression analysis, the correlation between above three exterior window variables and the lighting comfort degree is studied, and the optimal design strategies for lighting environment of western office buildings in severe cold region are summarized. The results show that when the WWR is $0.2-0.3$, the daylight comfortable performance is the best, the $\mathrm{WH}$ is about 0.9 and $\mathrm{W} / \mathrm{H}$ is between $0.4-0.8$, the office building has better daylight comfortable quality.
\end{abstract}

Keywords: Office Building, Lighting Comfort Performance, Window, Design Strategy

\section{光舒适导向的严寒地区办公建筑开窗设计策略研究}

王钊”，殷青

建筑学院, 哈尔滨工业大学, 哈尔滨, 中国

邮箱

1512391859@qq.com（王钊）, hityin@126.com（殷青）

摘要: 有效合理的天然采光能够营造舒适、健康的照明环境, 显著影响办公建筑室内光环境品质。合理的建筑形态设 计可以提高室内光环境质量和视觉舒适度，研究意义重大。本文结合严寒地区光气候特征，旨在分析窗墙比、窗台高 及窗宽高比比等外窗参数对办公建筑光环境舒适度的影响。以哈尔滨某办公建筑为例, 应用Rhino\&Grasshopper工具建 立参数化模拟模型; 采用控制变量实验方法, 利用DIVA工具, 计算不同窗墙比、窗台高及宽高比下的采光系数、全天 然采光百分百、有效天然采光照度和不舒适眩光概率四项光环境舒适性能评价数值; 总结归纳以光舒适性能为出发点 的严寒地区办公建筑开窗设计策略。结果表明, 窗墙比为 $0.2-0.3$ 时, 光舒适性能最佳, 窗台高为 0.9 左右, 窗宽高比在 0.4-0.8之间时, 办公建筑光舒适质量较好。

关键词: 办公建筑, 光舒适性能, 开窗, 设计策略 


\section{1. 引言}

随着城镇化水平的不断发展, 办公建筑的建设规模迅 速增长, 导致建筑立面造型设计丰盛而不注重建筑室内办 公人员光舒适性能的设计, 建筑的形态尺度和外窗的设计 只是作为建筑造型考虑的元素, 而其是否满足建筑室内工 作人员的作业要求, 是否满足工作人员的光舒适行性则考 虑的较少。舒适的办公空间光环境是安全、高效工作的基 础, 因此, 研究天然采光条件下办公建筑室内光舒适性能 具有现实意义。

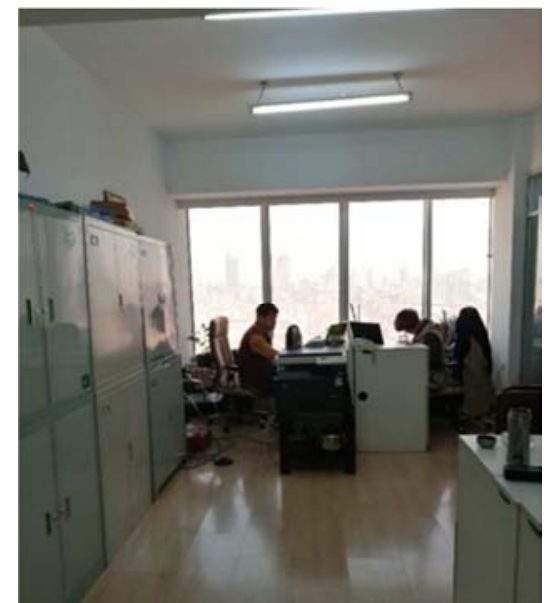

图1 办公楼室内现状。

\section{2. 光舒适性能}

\section{1. 光舒适性能定义}

国内外学者对光舒适的研究主要围绕采光量 (照度)、 光分布（光均匀性）、眩光分析、采光质量（显色性）四 方面来进行的（如图2），同时这四个方面涉及很多评价 指标。研究表明, 办公人员长期处于不舒适的光环境, 例 如过低的照度水平、不均匀的光分布、眩光现象严重等, 会对人体健康产生严重的伤害[1]。本文结合前人的研究成 果, 通过提出严寒地区办公建筑设计策略, 使办公建筑室 内既要保证满足一定的照度需求和采光均匀度的同时, 最 大限度的避免眩光的干扰。

\begin{tabular}{|c|c|c|c|}
\hline $\begin{array}{l}\text { 有效天然采光照度 } \\
\text { UDI }\end{array}$ & \multirow{6}{*}{ 采光均匀度 } & $\begin{array}{l}\text { 视赏舒适度 } \\
\text { VCP }\end{array}$ & \multirow{3}{*}{ 显色性 } \\
\hline \multirow{2}{*}{$\begin{array}{c}\text { 全天然采光百分比 } \\
\text { DA }\end{array}$} & & $\begin{array}{c}\text { 统一眩光值 } \\
\text { UGR }\end{array}$ & \\
\hline & & IIE标准眩光指数 & \\
\hline \multirow{2}{*}{$\begin{array}{c}\text { 采光系数 } \\
\text { DF }\end{array}$} & & \begin{tabular}{|l|} 
CGI \\
\end{tabular} & \multirow{3}{*}{ 色温 } \\
\hline & & $\begin{array}{c}\text { 不䂛适眩光指数 } \\
\text { DGI }\end{array}$ & \\
\hline $\begin{array}{c}\text { 照度 } \\
\text { I11 uninance }\end{array}$ & & $\begin{array}{c}\text { 不舒适眩光概率 } \\
\text { DGP }\end{array}$ & \\
\hline 采光量评价指标 & 北分布评价指标 & 眩光评价指标 & 采光质量评价指标 \\
\hline
\end{tabular}

图2 国内外光舒适主要评价指标。

\section{2. 光舒适性能评价指标}

(1) 采光系数 (Daylight Factor, DF)

采光系数由Waldram在1909年首次提出, 目前已被国 际照明委员会 (CIE) 认定为评价建筑空间天然采光性能 的基本指标 [2]。采光系数定义: “假设在CIE全阴天情况下, 室内给定平面内一点的照度与同一时刻室外无遮挡水平 面上的天空漫射光照度之比” [3]。

采光系数由于计算简单而被广泛应用, 但其对评价天 然采光性能存在两方面局限: 首先采光系数不能反映建筑 朝向变化; 其次, 采光系数是以全阴天天空为背景的, 未 考虑建筑地理位置的影响, 难免出现较大误差。因此, 目 前对于办公空间天然采光性能的研究, 采光系数已无法满 足建筑采光评价的要求。鉴于此，2006年Mardaljevic J在 CIBSE学术年会首次提出Climate-Based-Daylight-Modeling (CBDM), 即结合地域性光气候数据, 选择合适的天空 模型、精确地模拟平台以及使用的评价指标评价建筑天然 采光性能[4]。

（2）全天然采光百分比（Daylight Autonomy, DA）

全天然采光百分比最早在1989年瑞士标准中提出。 2001年, Reinhart F 和Walkenhorst $\mathrm{O}$ 将其重新定义作为动 态指标来评价建筑天然采光性能[5]。DA表示一年时间内, 工作面高度处满足最小天然采光照度 (3001x) 的时间与 工作总时间的比值 [6]。照明工程协会（Illuminating Engineering Society, IES)提出典型办公空间应满足DA值 在 $50 \%$ 以上[7]。相比DF, DA不局限于全阴天天空类型, 考虑了全年天空亮度分布的动态变化, 因而能够更准确地 评价全年办公空间天然采光质量。但DA只考虑了照度最 小值的情况, 忽视了过高的照度也会给室内工作人员带来 不舒适感。

(3) 有效天然采光照度 (Useful Daylight Illuminance, UDI)

2005年, Mardaljevic J 和Nabil A提出有效天然采光照 度, 是指一年中工作面高度处自然光的照度值在 1001x-20001x范围内的时间占工作总时间的百分比 [8] [9]。 UDI包括低于 $1001 x 、 100$ 至 $20001 x$ 之间和高于 $20001 x$ 三个评 价区间。IES规定UDI的最小标准值为 $50 \%$ 。UDI能够反映 不同照度区间内的自然光分布情况, 因其能独立评价 20001x以上的天然采光情况, 可以用于预测办公空间的眩 光情况。

考虑到采光系数已被纳入到国家标准, 且应用较为广 泛, 故仍选用其作为本文的天然采光性能评价指标, 同时 增加全天然采光百分比和有效天然采光照度两项动态指 标共同作为采光性能评价指标来评价严寒地区办公建筑 室内光舒适性能。 DGP )

（4）不舒适眩光概率（Daylight Glare Probability,

DGP是专用于评价天然采光条件下的不舒适眩光问 题, 而非人工照明光源的情景。基于计算机模拟计算, DGP 对于绝大多数天然采光条件下的眩光场景评价最准确, 因 此, 本文选用DGP为评价办公建筑室内的光舒适性能的指 标。 
针对不同眩光程度的得分范围, 将眩光程度划分为无 法察觉的眩光、可察觉的眩光、受干扰眩光和无法忍受的 眩光四种眩光程度。当 $\mathrm{DGP}<0.35$ 时, 为无法察觉眩光; $0.35<\mathrm{DGP}<0.4$ 时室内为可察觉眩光; $0.4<\mathrm{DGP}<0.45$ 时办 公建筑室内为受干扰的眩光; $D G P>0.45$ 时为无法忍受的 眩光。

\section{3. 模拟实验设计}

\section{1 . 模拟工具}

办公建筑光舒适性能参数化模拟需要技术模型支持, 本文结合Grasshopper参数化编程平台, 应用参数化编程技 术整合Rhinoceros建筑信息建模工具和Radiance、Daysim 建筑光环境性能模拟工具, 从而简化办公建筑光舒适性能
模拟的过程, 提高模拟工作效率。光舒适参数化模拟可结 合Grasshopper平台, 建立建筑形态空间参数模块, 三维形 态空间模型可在用Rhinoceros中有效显示，且模块中各建 筑形态空间信息具备自适应关联能力。

研究基于Python编程语言, 结合DIVA插件程序[10], 在Grasshopper平台中编写Radiance和Daysim光舒适性能 模拟工具与建模工具之间的数据交互模块, 实现性能模拟 工具与参数化建模工具之间的数据交互。

光舒适性能参数化模拟 (如图3) 实现了参数化建模 和采光性能模拟一体化, 提高了建筑光舒适性能模拟过程 自动化水平, 简化复杂模型的建模过程, 降低了人机交互 数据传输误差风险, 保证了模拟结果的可靠性, 能显著提 高建筑光舒适性能性能模拟效率和精度。

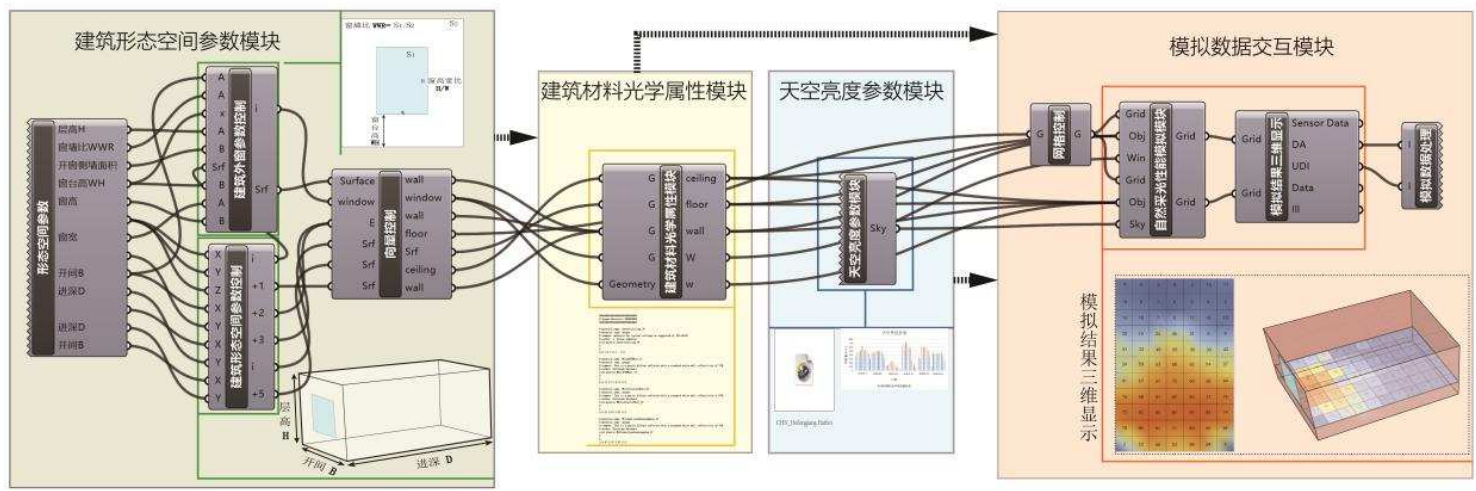

图3 严寒地区办公建筑光舒适性能参数化模拟模块。

\section{2. 模拟初始模型}

模拟实验首先运用严寒地区办公建筑光舒适参数化 模拟工具建立办公建筑初始模型，办公建筑模拟模型开间 $3.3 \mathrm{~m}$, 进深 $5.7 \mathrm{~m}$, 层高 $3.3 \mathrm{~m}$, 窗墙比设计初始值为 0.3 , 窗台高为 $0.9 \mathrm{~m}$, 外窗宽高比为 1 , 门位于内墙的中间位置,
高 $2.1 \mathrm{~m}$, 宽 $0.9 \mathrm{~m}$, 为普通木门。办公建筑内部有两张普通 深色办公桌, 长 $1.2 \mathrm{~m}$, 宽 $0.6 \mathrm{~m}$, 高 $0.8 \mathrm{~m}$, 其上分别有两台 电脑; 内部靠墙有三组浅色书柜, 长 $0.9 \mathrm{~m}$, 宽 $0.4 \mathrm{~m}$, 高 $1.85 \mathrm{~m}$ （如图4）。

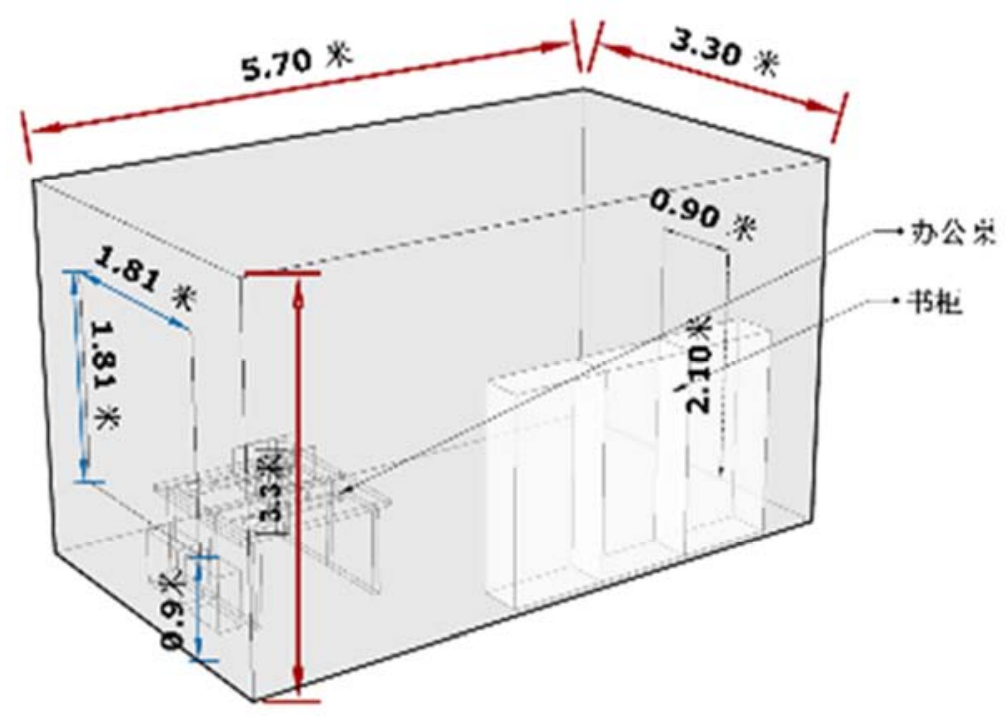

图4 初始模拟模型。 
研究建立形态空间几何模型是需要根据研究需求适 当简化几何模型, 已达到光学模拟计算的相关要求。详细 的模型可以产生视觉效果的真实感, 但可能某些构件对光 舒适性能的影响很小; 同时精细化的模型会影响计算机计 算速度，影响工作效率。

\section{3. 模拟参数设置}

办公建筑光舒适性能参数化模拟需要确定模拟分析 网格尺寸及环境模拟参数。下表为本本进行光舒适性能模 拟的的一些主要的模拟参数 [11], 如光线的反射的次数、 光分辨率等。DIVA中提供了简单的控制模块来调节这些 模拟参数值。模型采用CSWD哈尔滨地区典型气象年逐时 数据; 结合严寒地区办公建筑运行特征, 将模型中的运行
时间设定为周一到周五的8:00-17:00；根据工作面高度， 计算网格高度设定为距地面 $0.8 \mathrm{~m}$, 网格间距为 $0.5 \mathrm{~m}$ (如图 5 ）; 综合考虑模拟精度和耗时要求, 主要模拟精度参数 设置如表1所示。

办公建筑光舒适性能参数化模拟需要赋予模型材质, 主要为办公建筑室内墙体、地板、天花板、门以及家具的 反射率和外窗的透射率等光学属性。严寒地办公建筑室内 常见材料特性, 以白色墙体、白色天花板、浅白色地板、 双层外窗和深色木质办公桌为主, 并根据需要布置1 4组 书柜。通过对这些室内材料的光学属性进行实测, 得到墙 体和天花板的反射率为 0.8 , 地板反射率为 0.5 , 外窗透射 率为 0.66 , 办公桌的反射率为 0.369 , 书柜的反射率为 0.55 , 电脑显示器的反射率为 0.6 。

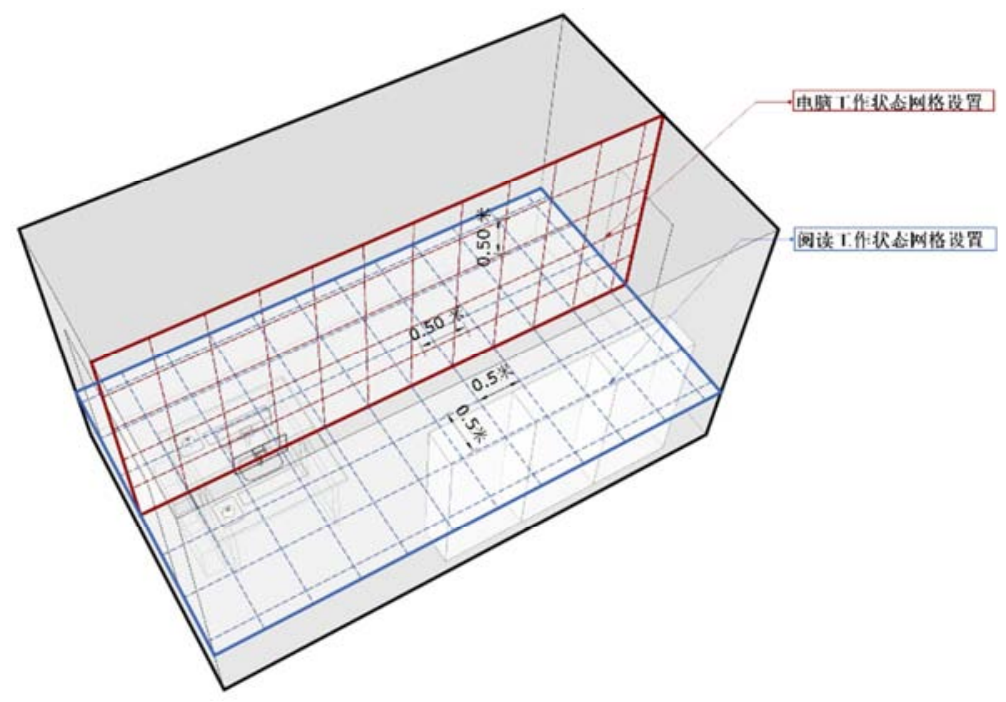

图5 初始模型网格设置。

表1 模拟精度参数设置。

\begin{tabular}{ll}
\hline 环境参数 & 数值 \\
\hline Ambient bounces & 2 \\
Ambient divisions & 1000 \\
Ambient super samples & 500 \\
Ambient resolution & 300 \\
Ambient accuracy & 0.1 \\
\hline
\end{tabular}

\section{4. 开窗设计策略}

\section{1 . 窗墙比设计策略}
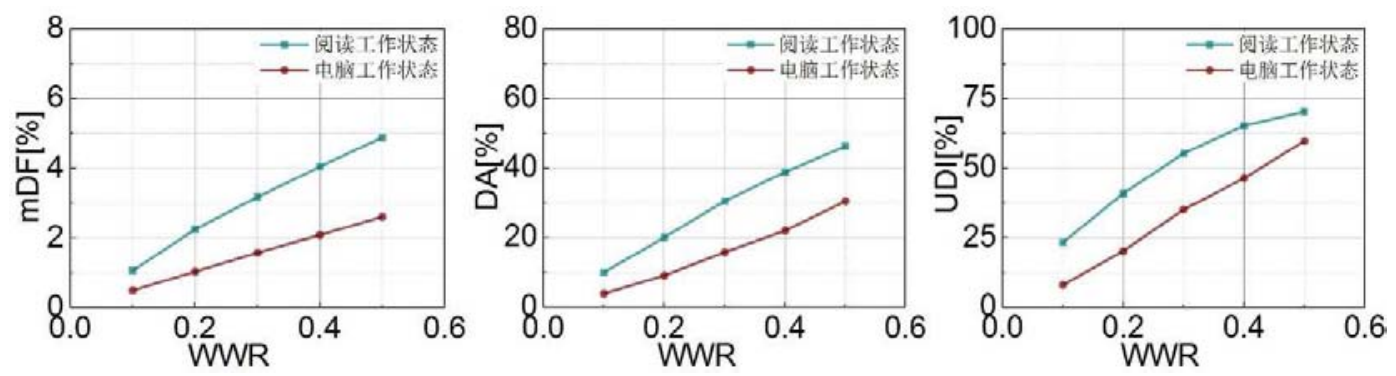

图6 建筑窗墙比与mDF、DA和UDI变化趋势图。 
图6为 $\mathrm{mDF} 、 \mathrm{DA}$ 和UDI随建筑窗墙比变化趋势图。由 图6可知, 建筑窗墙比对办公建筑室内平均采光系数、全 天然采光采光百分比和有效天然采光照度的影响较大; 两 种工作状态条件下的室内 mDF、DA和UDI值随建筑窗墙 比的增大呈现逐渐增大的变化趋势, 但窗墙比大于 0.4 时,
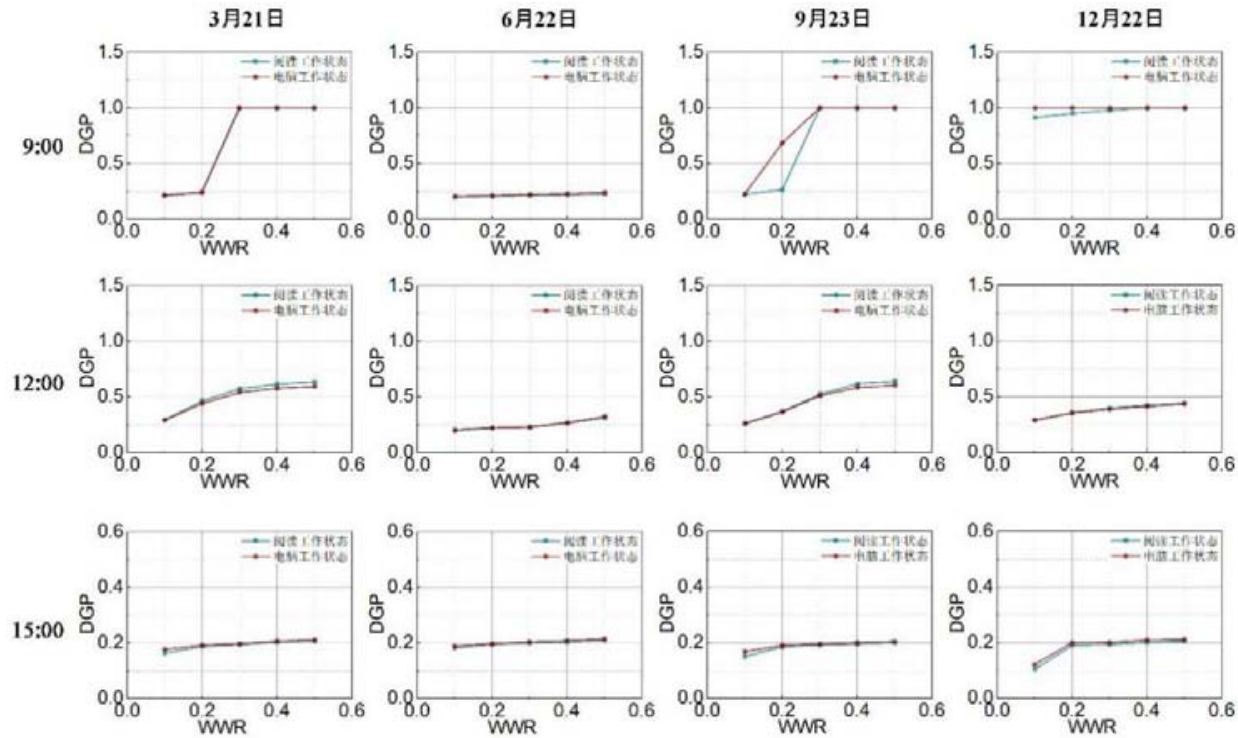

图7 建筑窗墙比与DGP变化趋势图。

上图7为春分、夏至、秋分和冬至日的建筑室内不舒 适眩光概率随建筑窗墙比变化的趋势图。可知建筑窗墙比 的变化对建筑室内DGP的影响较大; 不同季节不同时刻的 眩光程度存在显著差异; 无论窗墙比如何变化, 夏季建筑 室内均无眩光场景出现, 且全年下午 15:00左右为无法感 知的眩光; 其他季节时, 随着层高的增大, 眩光程度呈现 先增加后不变的趋势; 当窗墙比达到 0.3 后, 办公建筑室 内会出现令人无法忍受的眩光; 当窗墙比在 $0.1-0.2$ 之间时 眩光程度明显减小。

当窗墙比为 0.2 时, 秋季上午电脑工作状态的DGP值 明显高于阅读工作状态, 这是由于电脑工作状态的视角是
看向电脑，比阅读状态视角高，早上9:00刚好有直射光线 进入人的视野范围内，导致无法忍受的眩光出现。

因此, 在保持建筑外窗及其他形态空间参数不变的情 况下, 对于以单元式办公建筑为例的面积较小的办公空 间, 窗墙比在0.2-0.3之间可以实现室内光舒适性能较佳。

\section{2. 窗台高}

根据控制变量实验设计, 保证建筑其他形态空间参数 不变, 建筑窗台高在 $0.6 \mathrm{~m}-1.2 \mathrm{~m}$ 范围变化, 以 0.1 为步长整 理得建筑窗台高与 $m D F 、 D A 、 U D I$ 和DGP的变化关系。
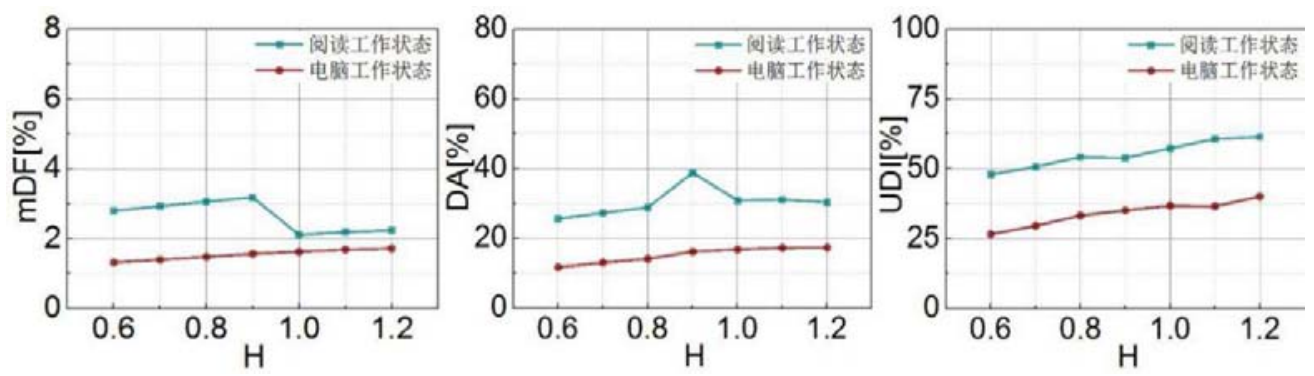

图8 建筑窗台高与mDF、DA和UDI变化趋势图。

图8为 $\mathrm{mDF} 、 \mathrm{DA}$ 和UDI随建筑窗台高变化趋势图。由 图可知, 建筑窗台高度对办公建筑室内平均采光系数、全 天然采光采光百分比和有效天然采光照度产生一定的影 响; 不同工作状态下 mDF、DA和UDI随建筑窗台高度变 化趋势显著不同; 阅读工作状态时, $\mathrm{mDF}$ 和DA随建筑窗 台高度的增长呈现先增大后减小的变化趋势, UDI则随窗
台高度的增长而持续缓慢增长, 当窗台高度超过 $0.9 \mathrm{~m}$ 后 $\mathrm{mDF}$ 和DA出现急速下降的现象, 产生这一现象的原因主 要是当窗台高度高于 $0.9 \mathrm{~m}$ 时, 近窗处的光线射入减少, 使 室内照度超过3001x的比例下降，导致DA值下降，同时照 度超过20001x的比例也降低, 使得UDI值增加; 电脑工作 
状态时 $\mathrm{mDF} 、 \mathrm{DA}$ 和UDI随建筑窗台高度的增加缓慢增长, 但当窗台高度达到 $0.9 \mathrm{~m}$ 后室内光舒适性能增长缓慢。

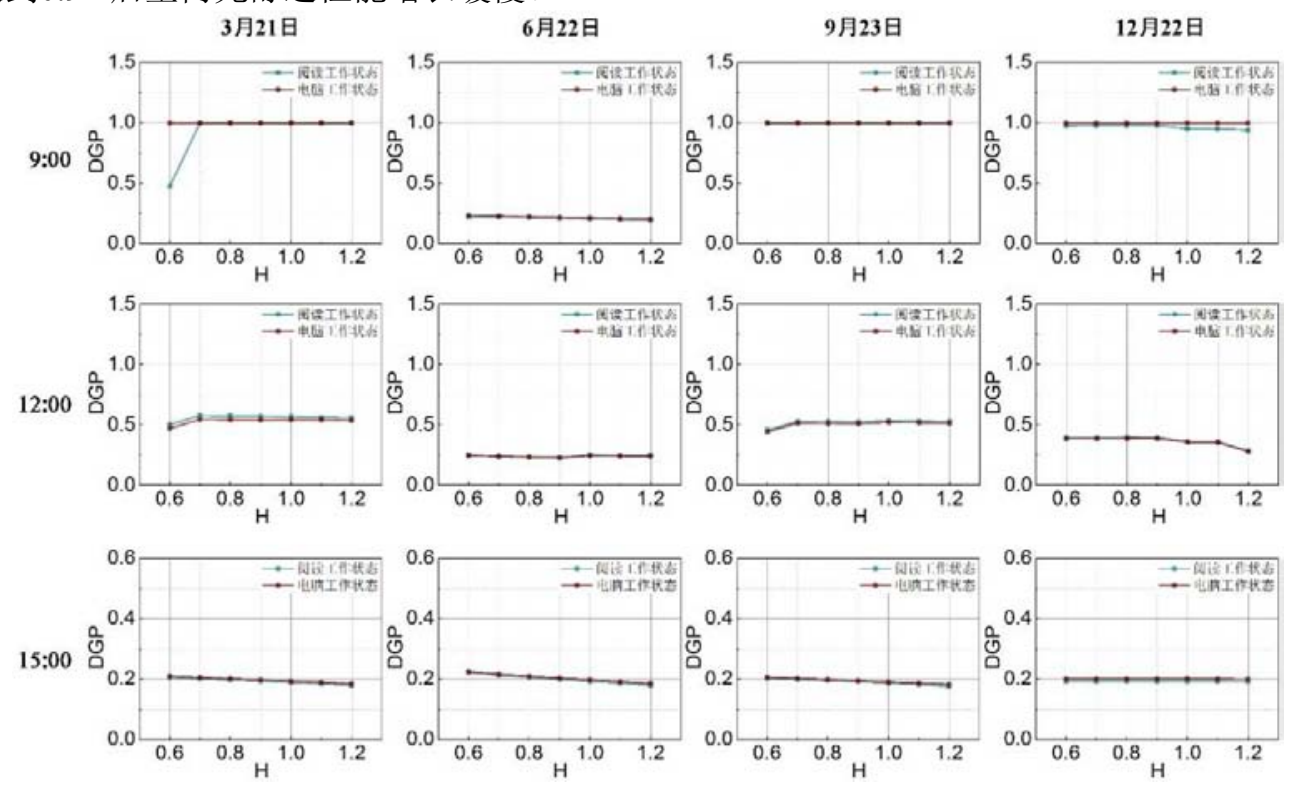

图9 建筑窗台高与DGP变化趋势图。

上图9为春分、夏至、秋分和冬至日的建筑室内不舒 适眩光概率随建筑窗台高度变化的趋势图。可知建筑窗台 高度的变化对建筑室内DGP的影响较小; 不同季节不同时 刻的眩光程度存在显著差异。夏季建筑室内DGP值均始终 小于 0.35 , 没有眩光场景出现, 且全年的下午 $15: 00$ 左右室 内人员感受不到眩光; 春季、秋季和冬季的上午建筑室内 DGP的值始终在 1 左右, 即室内总会出现无法忍受的眩光, 与窗台高度无关; 春季和秋季的中午随着窗台高度的增加 DGP先增加后不变的趋势, 但DGP的值始终大于 0.45 , 室 内存在无法忍受的眩光情况; 冬季的中午随着窗台高度的 增加, DGP值逐渐下降, 窗台高 $1.2 \mathrm{~m}$ 时室内降低至无法感 知的眩光; 两种工作状态同时刻的眩光程度无明显差异。 因此, 单独改变建筑窗台高度无法改善室内眩光程度。
因此，在保持建筑形态空间参数不变的情况下，以建 筑窗台高为实验变量时, 办公建筑光舒适性能波动较小; 窗台高度 $0.9 \mathrm{~m}$ 可以实现室内光舒适性能较佳, 但效果并不 显著; 在进行严寒地区办公建筑窗台高度设计时, 在考虑 办公空间内部光舒适性能的同时也需兼顾通风和视觉舒 适度的要求。

\section{3 . 窗宽高比}

根据控制变量实验设计, 保证建筑其他形态空间参数 不变, 建筑窗宽高比在 $0.2-1.6$ 范围变化, 以 0.2 为实验步长 整理得窗墙比与 $m D F 、 D A 、 U D I$ 和DGP的变化关系。
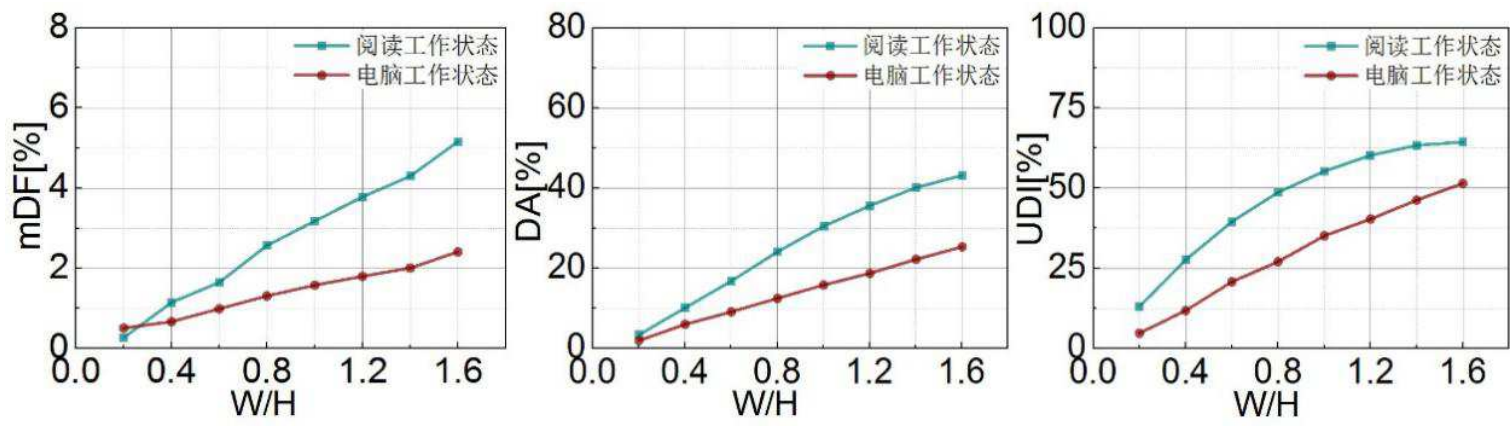

图10 窗宽高比与mDF、DA和UDI变化趋势图。

图10为 $\mathrm{mDF} 、 \mathrm{DA}$ 和UDI随建筑窗宽高比变化趋势图。 由图可知, 建筑窗宽高比对办公建筑室内平均采光系数、 全天然采光采光百分比和有效天然采光照度产生一定的 影响; 两种工作状态下 mDF、DA和UDI随建筑窗宽高比 变化趋势相似。阅读工作状态时, $\mathrm{mDF} 、 \mathrm{DA}$ 和UDI 随窗 宽高比的增大而增大, 但当窗宽高比达到1.2后, UDI增长
明显缓慢, 说明当窗宽高比达到 $1.2 \mathrm{~m}$ 后, 室内的光舒适性 能不再改善。

图11为春分、夏至、秋分和冬至日的建筑室内不舒适 眩光概率随建筑窗宽高比变化的趋势图。可知建筑窗宽高 比的变化对建筑室内DGP的影响较大; 不同季节不同时间 段内的眩光程度存在显著差异。 
由图可知, 夏季建筑室内DGP值均始终小于 0.35 , 没 有眩光场景出现, 且全年的下午 $15: 00$ 左右室内人员感受 不到眩光; 当窗宽高比达到 0.8 后, 春季和秋季的建筑室 内DGP的值迅速增加至1左右, 即室内总会出现无法忍受 的眩光; 而冬季上午当窗宽高比为 0.4 时即开始出现眩光。 因此, 保持建筑窗宽高比在0.4-0.8内时, 可以有效改善室 内的眩光情况。
因此，在保持建筑形态空间参数不变的情况下，以建 筑窗宽高比为实验变量时, 办公建筑光舒适性能波动较 大；窗宽高比在0.4-0.8 可以实现室内光舒适性能最佳; 但 在设计外窗尺寸时, 考虑到窗宽高比较小时, 外窗宽度较 小, 影响室内人员的空间舒适性和心理感受, 因此在进行 严寒地区办公建筑窗宽高比设计时, 需兼顾心理感受和视 觉舒适度的要求。
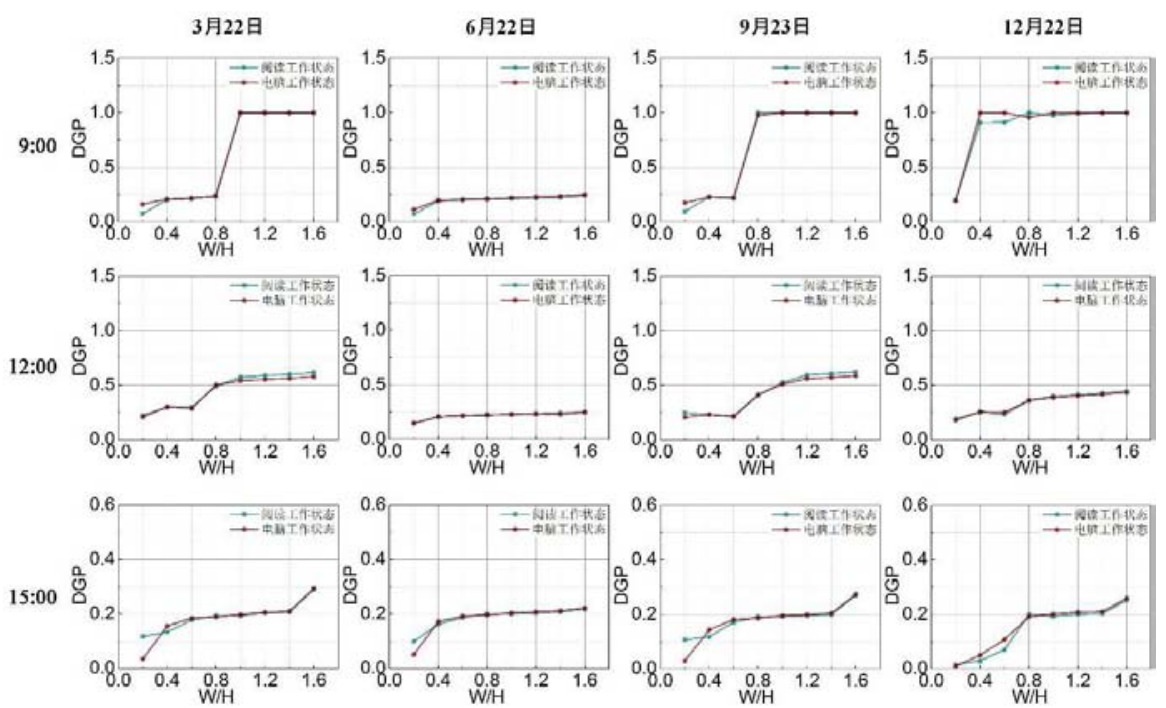

图11 建筑窗宽高比与DGP变化趋势图。

\section{5. 结论}

本文提出了严寒地区办公建筑光舒适性能影响下的 开窗设计策略。严寒地区办公建筑光舒适性能设计策略以 办公建筑室内不舒适眩光概率、平均采光系数、全天然采 光百分比、有效天然采光照度为设计目标; 以建筑窗台高、 窗墙比、窗宽高比为设计参量。得出层高对办公建筑室内 光舒适性能影响较小, 窗墙比为0.2-0.3 时, 光舒适性能最 佳, 窗台高为 0.9 左右, 窗宽高比在 $0.4-0.8$ 之间时, 办公建 筑光舒适质量较好。

\section{致谢}

本文受国家自然科学基金面上项目 (51578172) 资助。

\section{参考文献}

[1] Boyce P R. Human Factors in Lighting, Second Edition [J]. Crc Press, 2003.

[2] CIE. International lighting vocabulary. Commission Internationale de l'Eclairage [J]. CIE, 2011.

[3] 刘加平. 建筑物理[M]. 北京: 中国建筑工业出版社, 2009: 37-52。
[4] Kazanasmaz T, Grobe L O, Bauer C, et al. Three approaches to optimize optical properties and size of a South-facing window for spatial Daylight Autonomy [J]. Building and Environment, 2016, 102:243-256.

[5] Reinhart C F, Walkenhorst O. Validation of dynamic RADIANCE-based daylight simulations for a test office with external blinds [J]. Energy and Buildings, 2001, 33(7):683-697.

[6] Reinhart C F, Mardaljevic J, Rogers Z. Dynamic Daylight Performance Metrics for Sustainable Building Design [J]. Leukos the Journal of the Illuminating Engineering Society of North America, 2013, 3(1-4):7-31.

[7] Heschong L, Wymelenberg V D, (Vice Chair), Keven, et al. Approved Method: IES Spatial Daylight Autonomy (sDA) and Annual Sunlight Exposure (ASE) [S]. Illuminating Engineering Society, 2016.

[8] Nabil A. Useful daylight illuminance: a new paradigm for assessing daylight in buildings [J]. Lighting Research and Technology, 2005, 37(1):41-59.

[9] Nabil A, Mardaljevic J. Useful daylight illuminances: A replacement for daylight factors [J]. Energy and Buildings, 2006, 38(7):905-913.

[10] Reinhart C, Lagios K., Niemasz J. 2011. DIVA for Rhino Version 2.0. http://www.diva-for-rhino.com/.

[11] 赵蓓, 余庄. 建筑光环境的计算机模拟设计-阅览室光环境 的计算机模拟与实测分析. 中国建筑学会, 2003年学术年会 论文集，2003。 